\title{
Conversation Analysis for Facilitation in Children's Intercultural Collaboration
}

\author{
Mizuki Motozawa \\ Ritsumeikan University \\ Kusatsu, Japan \\ is0380sr@ed.ritsumei.ac.jp
}

\author{
Yohei Murakami \\ Ritsumeikan University \\ Kusatsu, Japan \\ yohei@fc.ritsumei.ac.jp
}

\author{
Mondheera Pituxcoosuvarn \\ Ritsumeikan University \\ Kusatsu, Japan \\ mond-p@fc.ritsumei.ac.jp
}

\author{
Toshiyuki Takasaki \\ NPO Pangaea \\ Kyoto, Japan \\ toshi@pangaea.org
}

\author{
Yumiko Mori \\ NPO Pangaea \\ Kyoto, Japan \\ yumi@pangaea.org
}

\begin{abstract}
In order to find solutions to various international problems, Global Citizenship Education (GCED) for children must consider the diversity of societies beyond basic issues of language and culture. For GCED, machine translation can be used as a tool to allow children to collaborate without a common language. However, low-resource language speakers often cannot enter the conversation and have difficulties in participating in a collaboration, because existing machine translations have poor translation quality in the low-resource languages. Messages from facilitators play an important role in encouraging children's responses and participation. We, therefore, have analyzed the role of the facilitator in a real-world intercultural children workshop. Specifically, we examine actual conversation $\log$ data that links the facilitator's utterances with children's utterances in adjacency pairing. We annotate the paired data with tags and then statistically analyze the tagged data. The analysis results show that some types of facilitator messages can significantly impact the responses of low-resource language speaking children. For example, "request" type utterances tended to encourage responses.
\end{abstract}

\section{CCS CONCEPTS}

- Human-centered computing $\rightarrow$ Empirical studies in collaborative and social computing.

\section{KEYWORDS}

intercultural collaboration, multilingual communication, machine translation

\section{ACM Reference Format:}

Mizuki Motozawa, Yohei Murakami, Mondheera Pituxcoosuvarn, Toshiyuki Takasaki, and Yumiko Mori. 2021. Conversation Analysis for Facilitation in Children's Intercultural Collaboration. In Interaction Design and Children (IDC '21), June 24-30, 2021, Athens, Greece. ACM, New York, NY, USA, 7 pages. https://doi.org/10.1145/3459990.3460721

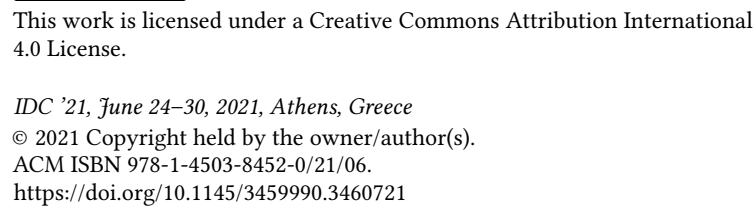

This work is licensed under a Creative Commons Attribution International 4.0 License.

IDC '21, June 24-30, 2021, Athens, Greece

(C) 2021 Copyright held by the owner/author(s)

ACM ISBN 978-1-4503-8452-0/21/06.

https://doi.org/10.1145/3459990.3460721

\section{INTRODUCTION}

GCED is built around three key principles:(1) respect for cultural, linguistic, and ethnic diversity, (2) solidarity, and (3) a shared sense of humanity, which distinguish it from other educational approaches. To establish these principles in childhood, intercultural collaboration using machine translation for children has become more popular. KISSY (Kyoto International Summer School for Youth) is one of the workshops where children from different countries can engage in group activities, which is annually held by NPO Pangaea, a non-profit organization. This workshop groups the children in intercultural teams with one adult facilitator for each team to support them. Communication in a foreign language is more difficult than communication in a native language [7]. Therefore, a machine translation embedded tool is used to support communication among children speaking different languages. However, children speaking low-resource languages have difficulties in understanding the conversation and expressing their intended meaning with their own words because the translation quality of low-resource languages is relatively poor. As a result, some children are less talkative than others, because the children with low-resource languages cannot follow the conversation [4]. An existing study found that questions from the adult facilitator can support children group work; they examined a design task with guided children's dialogs [8]. Hence, we focus on the behavior of the adult facilitator, who is considered to be important in guiding the children's conversation in collaborative settings. On particular note, this paper pays particular attention to the type of facilitator speech and analyzed chat dialogs between a facilitator and children to clarify which utterances increased the children's responses. To this end, we collected conversation log data from an international workshop by children and linked the facilitator's utterances with children's utterances in adjacency pairing. We then annotated the paired data with tags defined on the speech-act verbs by Vanderveken [9]. We then identified the significantly effective utterances of the facilitator using $\chi^{2}$ tests and residual analysis.

This paper first introduces related studies and research and explains the conversation data analyzed. After that, it defines tags, annotates the conversation data with the tags and performs statistical tests on the tagged data. Last, the analysis results are discussed, and some conclusions are drawn. 


\section{INTERCULTURAL COLLABORATION USING MACHINE TRANSLATION}

In intercultural collaboration, machine translation allows people who cannot speak a common language to work together, especially children. However, machine translation causes some problems, including translation errors and the difficulty of understanding each other [10]. Furthermore, misspellings of words can lead to misunderstandings of messages, making mutual understanding even more difficult [1]. These translation errors may sometimes convey the messages in a meaning that the speaker did not intend, and the message is misunderstood. One of the most important problems is that child speakers of low-resource languages cannot understand messages translated from other languages and the other language speakers cannot understand messages translated from low-resource languages because the translation quality in low-resource languages is quite low compared to rich resource languages such as English. This results in child speakers of the low-resource language having difficulties in communicating their intended contents to others [11].

For this study, we collected conversation log data of KISSY (Kyoto International Summer School for Youth) held by NPO Pangaea [5]. This summer school consisted of 28 children from Japan, Kenya, Cambodia and Georgia, 8 to 15 years old and adult facilitators. Facilitators who participate here have attended two types of training programs organized by the NPO including a general facilitator training for Pangaea activities and a special training for the KISSY workshop. The training contents includes how to interact with children, safety management, KISSY implementation flow, and how to use KISSY tool. The facilitators were also provided with a list of activities with detailed time schedules. The participants were divided into four groups consisting of seven children and one facilitator such that nationality and language were not biased. As shown in Figure 1, each team worked on issues defined in the SDGs (Sustainable Development Goals) $^{1}$. Most conversations between team members were conducted using a multilingual chat tool (Figure 2) accessed via the Language Grid [3], which translates sender's messages into the languages of the other children so that all the

${ }^{1}$ https://www.un.org/sustainabledevelopment/

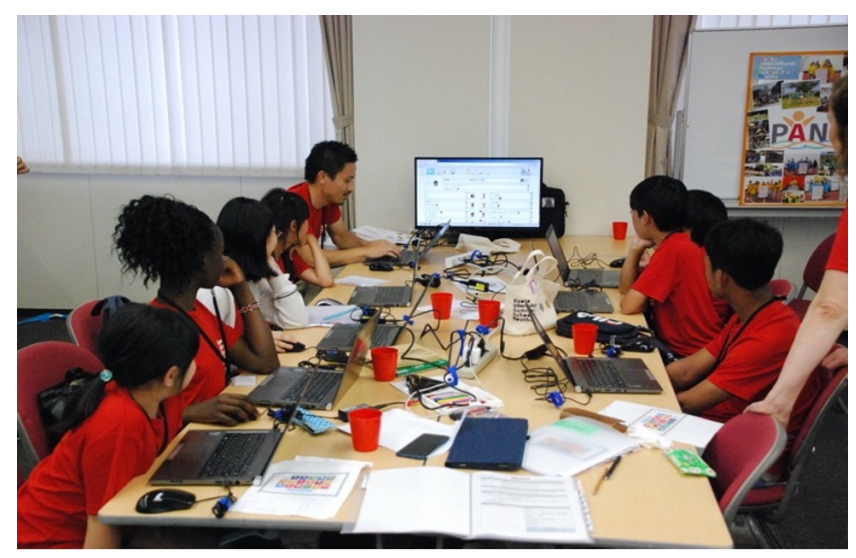

Figure 1: Atmosphere of group work (Photo offered by NPO Pangaea)

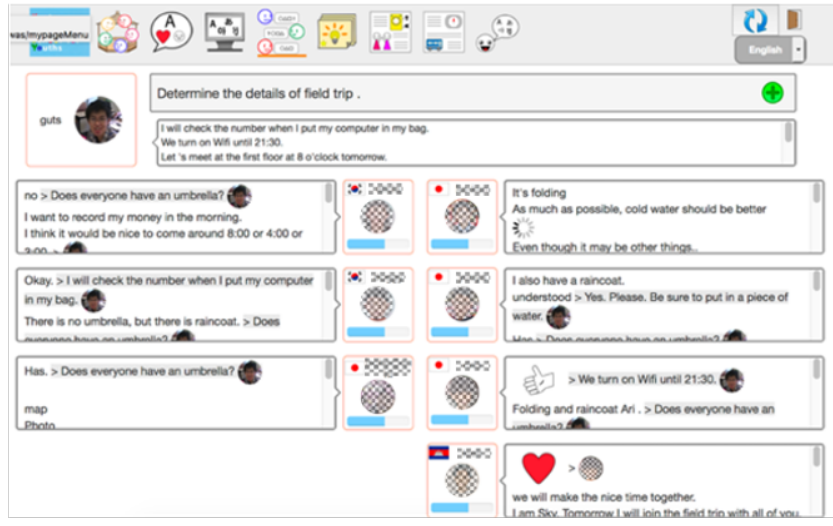

Figure 2: Chat tool UI (Photo offered by NPO Pangaea)

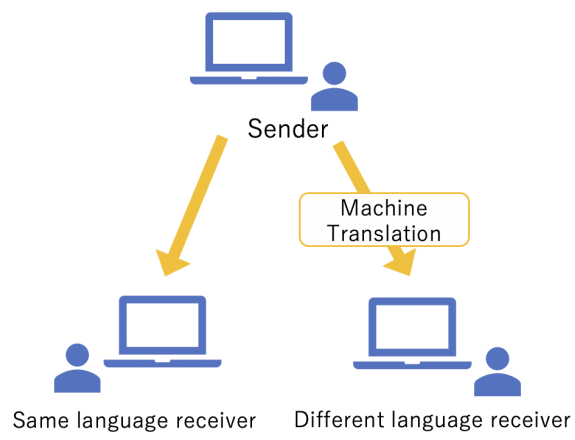

Figure 3: Communication channel of the chat tool

team members could understand the messages. Although verbal communication is allowed, even children speaking the same languages often communicate with each other using the tool to share the conversation with other members as shown in Figure 3. The children spent three days making a clay animation that explained their solution to the SDGs issues. The first two days focused on discussion to decide a SDGs issue to be addressed by each team and to design a story of the animation. After that, each group had been producing the clay animation until the third day. We could gather and analyze about seven hours of each team's communication of group work from the first and second days. The nationalities of the participants in each team are listed in Table 1. The target lowresource language in the present analysis is Khmer, spoken by the Cambodian children in Team A and Team C.

\section{DATA PREPARATION}

\subsection{Data Formatting}

First, message contents, utterer, utterance language and utterance time were extracted from conversation log data. The messages that were not necessary for discussion, such as meaningless strings and meaningless consecutive posting of emoji functions [2] were removed. 
Table 1: Nationality breakdown of team members

\begin{tabular}{|c|c|c|c|c|c|}
\hline & & Japanese & Kenyan & Georgian & Cambodian \\
\hline \multirow{2}{*}{ Team A } & $\begin{array}{l}\text { Facilitator } \\
\text { (Language) }\end{array}$ & & & $\begin{array}{c}1 \\
\text { (Georgian) }\end{array}$ & \\
\hline & $\begin{array}{l}\text { Children } \\
\text { (Language) }\end{array}$ & $\begin{array}{c}5 \\
\text { (Japanese) }\end{array}$ & $\begin{array}{c}1 \\
\text { (English) }\end{array}$ & & $\begin{array}{c}1 \\
\text { (Khmer) }\end{array}$ \\
\hline \multirow{2}{*}{ Team B } & $\begin{array}{l}\text { Facilitator } \\
\text { (Language) }\end{array}$ & $\begin{array}{c}1 \\
\text { (English) }\end{array}$ & & & \\
\hline & $\begin{array}{l}\text { Children } \\
\text { (Language) }\end{array}$ & $\begin{array}{c}5 \\
\text { (Japanese) }\end{array}$ & $\begin{array}{c}1 \\
\text { (English) }\end{array}$ & & $\begin{array}{c}1 \\
\text { (English) }\end{array}$ \\
\hline \multirow{2}{*}{ Team $C$} & $\begin{array}{l}\text { Facilitator } \\
\text { (Language) }\end{array}$ & & $\begin{array}{c}1 \\
\text { (English) }\end{array}$ & & \\
\hline & $\begin{array}{l}\text { Children } \\
\text { (Language) }\end{array}$ & $\begin{array}{c}5 \\
\text { (Japanese) }\end{array}$ & & $\begin{array}{c}1 \\
\text { (English) }\end{array}$ & $\begin{array}{c}1 \\
\text { (Khmer) }\end{array}$ \\
\hline \multirow{2}{*}{ Team D } & $\begin{array}{l}\text { Facilitator } \\
\text { (Language) }\end{array}$ & & & & $\begin{array}{c}1 \\
\text { (English) }\end{array}$ \\
\hline & $\begin{array}{l}\text { Children } \\
\text { (Language) }\end{array}$ & $\begin{array}{c}5 \\
\text { (Japanese) }\end{array}$ & & $\begin{array}{c}1 \\
\text { (English) }\end{array}$ & $\begin{array}{c}1 \\
\text { (English) }\end{array}$ \\
\hline
\end{tabular}

\subsection{Utterance Pairing}

Adjacency pairs were recognized in the formatted data by linking the utterances of the facilitator and children. Table 2 shows the number of adjacent response pairs, including no-responses, made by Khmer speaking children of Team A and Team $C$, and the response rate. In addition, the data for all children, excluding the Khmer speaking children, are also shown. There were more utterances made by Team $C$ facilitator than Team $A$ facilitator, while Team $A$ facilitator received more responses from the Khmer speaking child than Team $C$ facilitator. From this data, we could assume that the Team $A$ facilitator might had made more effective utterances in terms of encouraging responses by the Khmer speaking children because the Khmer speaking child of Team A exhibited a higher response rate than even children of other languages. To clarify what type of facilitator utterance was effective, we categorized the facilitator's utterances based on speech-act theory and counted the number of responding utterances in each classification.

\section{ANNOTATION OF UTTERANCE}

To analyze the data, the facilitator utterances in each of the four teams were classified based on Searle's speech categories [6]. We tagged each utterance with Searle's speech categories and Vanderveken's speech act verbs, which are classified as a subcategory of Searle's.

\subsection{Tag Definition}

Searle's five types of speech are shown in Table 3. In this classification, the speech that prompts the child to express some opinions or ideas is "directives", which leads to the child's responses. For this reason, we used Vanderveken's verbs of speech act, which were defined as a subdivision of this "directive" category. Using this classification, we analyzed what kind of directive speech is effective for children. The simplest type of the action-directed verbs is "direct". With special conditions to this verb, we could derive other verbs of speech act: "request", "tell", "insist", and "suggest". This tag set definition is shown in Table 4. The first author annotated facilitators' utterances with this tag set. We then summed up the number of annotated facilitator utterances and the number of responses by Khmer speaking children to the facilitator's utterances. Furthermore, to compare a characteristic feature of the Khmer speaking children and other languages speaking children, data of all children's responses excluding the Khmer speaking children were collected and summed up. Table 5 shows the number of utterances by each facilitator in each category, and Table 6 shows the number of responses by Khmer speaking children and the number of responses by other children in each team.

\section{DIALOG ANALYSIS}

\subsection{Test of Independence}

To analyze whether the verbs of speech act used by the facilitator changes the number of responses of Khmer speaking children and other language children, we conducted the $\chi^{2}$ test using the tabulated data of the number responses and no-responses from Khmer speaking children and the other language speaking children for each classification.

The result of the test is shown in Table 7. For the children other than Khmer, there was no significant difference in the results for the three teams other than Team C. In other words, in most cases, the way in which the facilitator gave the instructions had no effect on the number of other language speaking children's responses. On the other hand, for the low-resource language children, there was a significant difference for the children in Team A, but not in Team C. From this result, Team A's facilitator's verbs of speech act increased the number of responses from low-resource language children.

\subsection{Residual analysis using adjusted residual}

To analyze which verb of speech act made a significant difference, we carried out a residual analysis on the Table 6 . Specifically, we 
Table 2: Number of responses and response rate of Khmer speaking children

\begin{tabular}{rccc}
\hline & \multicolumn{2}{c}{ Khmer speaking children } & Other language speaking children \\
& Team A & Team C & 270 \\
\hline Number of responses & 10 & 7 & 1088 \\
Number of no-responses & 21 & 84 & $20 \%$ \\
Response rates & $40 \%$ & $8 \%$ & \\
\hline
\end{tabular}

Table 3: Searle's five classifications of speech types

\begin{tabular}{|c|c|}
\hline Classification & Description \\
\hline \multirow{2}{*}{ Assertives } & Describes the state of things. \\
\hline & Ex. "We need to talk about $\sim . "$, "We need to prepare a presentation." \\
\hline \multirow{2}{*}{ Commisives } & Binds the speaker to a future action. Promises a future action. \\
\hline & Ex. "We will now discuss the story of clay animation.", "After the break, we will talk about ." \\
\hline \multirow{2}{*}{ Directives } & To try to make the listener do something. \\
\hline & Ex. "Tell me what you think.", "What is your opinion about ?" \\
\hline \multirow{2}{*}{ Expressives } & Simply expresses the speaker's feelings. \\
\hline & Ex. "That thing is very interesting!" \\
\hline Declarations & $\begin{array}{l}\text { To carry out an action in the present by saying that the speaker is carrying the action. } \\
\text { Ex. "This concludes our discussion for today." }\end{array}$ \\
\hline
\end{tabular}

Table 4: Vanderveken's verbs of speech act, which are defined as a subdivision of Searle's category

\begin{tabular}{|c|c|}
\hline Classification & Description \\
\hline \multirow{3}{*}{ Request } & Give instructions in a way that gives the listener the right to veto them. \\
\hline & Give the instructions carefully. \\
\hline & Ex. "Do you have an opinion about ?", "Have you got some ideas about ?". \\
\hline \multirow{3}{*}{ Tell } & Give the instructions in a way that leaves no room for refusal. \\
\hline & The instructions are based on the assumption that the listener will respond. \\
\hline & Ex. "What do (name) think?", "Tell us what you think." \\
\hline \multirow{3}{*}{ Insist } & Persistently gives instructions. \\
\hline & Saying the same thing again in the same way that he has already said it. \\
\hline & Ex. "What is your opinion about $\sim$ ?(Say the same thing as at the beginning.)" \\
\hline \multirow{3}{*}{ Suggest } & The weak attempt to get the listener to perform an action, \\
\hline & regardless of whether or not the speaker rejects the instruction. \\
\hline & Ex. "If anyone wishes to change his or her opinion about the mission, please do so.' \\
\hline
\end{tabular}

Table 5: Number of facilitator's utterances in each category

\begin{tabular}{rcccc}
\hline & Team A & Team B & Team $C$ & Team D \\
\hline Request & 9 & 9 & 22 & 20 \\
Tell & 5 & 4 & 19 & 4 \\
Insist & 4 & 3 & 6 & 3 \\
Suggest & 2 & 2 & 1 & 0 \\
\hline Total & 20 & 18 & 48 & 27 \\
\hline
\end{tabular}

used the following formula to calculate the adjusted residuals for each speech act verb from the total number of responses and no responses, respectively, and the values are compared.

$$
d_{i j}=\frac{f_{i j}-E_{i j}}{\sqrt{E_{i j}\left(1-\frac{r_{i}}{n}\right)\left(1-\frac{c_{j}}{n}\right)}}
$$

\subsection{Comparative analysis of the children's responses to facilitator's utterance}

From the results of the calculated residual values, verbs of speech act with residuals greater than the expected value $(=1.96)$ tend to elevate the child's responses. On the other hand, the verbs of speech act with residuals less than the expected value $(=-1.96)$ were linked to the child's lack of response. We conducted this analysis on the data of the low-resource language in Team A, and all the children except the low-resource language in Team $C$. 
Table 6: Number of responses to facilitator's utterances in each category

\begin{tabular}{rcc|cccc}
\hline & \multicolumn{5}{c}{ Khmer children } & \multicolumn{4}{c}{ Other children } \\
& Team A & Team C & Team A & Team B & Team C & Team D \\
\hline Request & 8 & 3 & 23 & 12 & 36 & 61 \\
Tell & 0 & 1 & 11 & 8 & 16 & 6 \\
Insist & 2 & 2 & 6 & 1 & 4 & 8 \\
Suggest & 0 & 0 & 7 & 1 & 2 & 0 \\
\hline Total & 10 & 6 & 47 & 22 & 58 & 75 \\
\hline
\end{tabular}

Table 7: Results of $\chi^{2}$ test $\left({ }^{*}: p<0.05,{ }^{* *}: p<0.01\right.$, ns: $\operatorname{not}$ significant)

\begin{tabular}{ll|cc}
\hline & & $p$-value & $\chi^{2}$ value \\
\hline \multirow{2}{*}{ Khmer children } & Team A & $.006^{* *}$ & 10.230 \\
& Team C & $.190 \mathrm{~ns}$ & 4.757 \\
\hline \multirow{4}{*}{ Other children } & Team A & $.239 \mathrm{~ns}$ & 4.216 \\
& Team B & $.116 \mathrm{~ns}$ & 5.892 \\
& Team C & $.025^{*}$ & 9.291 \\
& Team D & $.090 \mathrm{~ns}$ & 4.804 \\
\hline
\end{tabular}

Table 8: Results of the calculated residual values

\begin{tabular}{rcc}
\hline & $\begin{array}{c}\text { Khmer child } \\
\text { Team A }\end{array}$ & $\begin{array}{c}\text { All children excluding Khmer child } \\
\text { Team C }\end{array}$ \\
\hline Request & $\mathbf{3 . 1 4 6}$ & $\mathbf{2 . 7 7 7}$ \\
Tell & $-\mathbf{2 . 5 8 2}$ & $\mathbf{- 2 . 0 9 1}$ \\
Insist & 0 & -1.444 \\
Suggest & -1.491 & 0.814 \\
\hline
\end{tabular}

Table 8 shows the calculated adjusted residuals for Khmer child in Team $A$ and all children except Khmer child in Team C. The results show that, for low-resource language speaking children in Team A and the children speaking high-resource language in Team $C$, "request" utterances boosted the children's responses, while "tell" utterances inhibited the children's responses.

\section{DISCUSSION}

Team $A$ and Team $C$ showed significant differences for the number of responses from low-resource language child and high-resource language children, respectively. To investigate the reasons, we focused on the number of facilitator utterances and the message content of the utterances in the categories with significant differences (Table 8).

\subsection{Quantitative comparison of facilitators' utterances}

When the number of utterances of each facilitator in Table 5 was compared using $\chi^{2}$ test, there was no significant difference in the number of utterances compared to one from the other teams. This result does not represent the facilitators in Team A and Team C tended to employ extremely more "request" utterances and extremely less "tell" utterances than the other teams' facilitators. That is, the number of utterances of facilitators does not cause significant differences for the number of responses from low-resource language child and high-resource language children. Since it is difficult to observe the differences in the quantity of the triggers of responses from the children, the reason is considered to be the quality of the utterances of the facilitators.

\subsection{Qualitative comparison of facilitator's utterances}

6.2.1 "Request" utterances. "Request" utterances produced a significant difference in the high-resource language children's responses in Team C. In this team, the facilitator asked several close-ended questions. As a consequence, the children responded easily, and the number of responses was high. However, close-ended questions do not encourage children to express their own opinions, which is important in this type of workshop. Therefore, it is necessary to analyze the effective "request" utterances that encourage children to respond with their own opinions.

Regarding the results of the low-resource language children, there was a significant difference in the number of responses of the low-resource language children of Team A, but there was no significant difference in the number of responses of the low-resource language children of Team C. In order to understand this result, we compared the message content of the facilitators of these two teams. The facilitator of Team A started the discussion after presenting the content of the upcoming discussion in advance, such as "Let's discuss the roles now". On the other hand, the facilitator of Team $C$ did not define the content of the discussion beforehand and started the discussion with "Who would like to be a sound creator?" without defining the content of the discussion beforehand. Moreover, the facilitator of Team A issued "request" utterance several times. There were many cases in which low-resource language speaking children responded well to this repetitive usage of "request". The low-resource language child got a translation result for each of the repeated utterances. These translation results would have helped the participants to understand the facilitator's intentions. It is necessary to consider not only the one-to-one dialogue but also the combination of facilitator utterances as a temporal sequence, although only the response by neighboring utterance pairs was examined in this paper. On the other hand, looking at the responses of the low-resource child in Team $C$, five out of the six responses were either "I understand" or responses in which she chooses a response option, suggesting that the children in Team $C$ did not fully 
Table 9: Examples of facilitator's speech verb of each speech act

\begin{tabular}{rll}
\hline \multirow{2}{*}{ Speech act } & \multicolumn{1}{c}{ Team A } & \multicolumn{1}{c}{ Team C } \\
\hline \multirow{2}{*}{ Tell } & "Do you use a PC or mobile at school?" & "(Name), do you have idea what sound?" \\
& "Please say which Pangaea Mission is good." & "(Name), what do you think?" \\
\hline \multirow{2}{*}{ Request } & "What are some ideas for solving my chosen Pan- & "Who can help with sculptor?" \\
& gaea Mission polls?" & \\
& "Do you have any questions about the solution & "Can we find a good solution for mission one?" \\
& written by team members?" & \\
\hline
\end{tabular}

understand the content of the discussion and therefore did not respond well to the facilitator's speech regardless of its classification. It is possible that the children in Team $C$ did not fully understand the content of the discussion and therefore did not respond to the facilitator's speech regardless of its category.

6.2.2 "Tell" utterances. The category of the utterance "tell" yielded a significant difference in the children's non-response. The facilitators of Team C mostly instructed particular children by addressing their names in "tell" utterances. Although name-calling utterances were observed in other categories, "tell" utterances were accompanied by children's name more than others. Also, this utterance was done equally for each child. Such utterances were almost always responded to only by the child to whom they were addressed. Therefore, the number of responses from the team in this category was low, which may have affected the results. When the same comparison was made using only the utterances that excluded the utterances that mentioned the child's name, no significant difference was found for "tell" utterances. We note that the facilitator of Team A made few name-calling utterances.

For the low-resource language speaking children in Team A, "request" utterances tended to promote the low-resource language speaking children to response. Whereas "tell" utterances tended to limit the responses. The actual log data is shown in the Table 9. From this example, it can be seen that "tell" utterances, which are utterances that do not give the listener the right of veto, such as "Do you use a PC or mobile at school?" or "Please say which Pangaea Mission is good", usually produced no response from the low-resource language children. On the other hand, "request" utterances such as "What are some ideas for solving my chosen Pangaea Mission polls?" or "Do you have any questions about the solution written by team members ?" allow low-resource children responded well as they gave some degree of veto power. This result is due to the psychological effect of "psychological reactance". This is the psychological tendency to react against a task when it is forced upon us. Since utterances with veto are less coercive, they may have suppressed the reaction against it.

\section{CONCLUSION}

This study investigated the issue of poor response rates from lowresource language speaking children in multilingual communication via machine translation embedded chat tool by classifying the facilitator's utterances in the chat log. Then, we compared the number of children's responses to each type of facilitator's utterances. We found that the number of responses from low-resource language children tended to increase or decrease depending on how the facilitator instructed them. Specifically, among the types of verbs of speech acts, "request" utterances were found to increase the response rates of low-resource language children. On the other hand, "tell" utterances tended to inhibit the response rates of lowresource children. In addition, repeating "request" utterance was effective in promoting responses from the low-resource language speaking children. Note that we have not been able to confirm the uniformity of the tag set used in this paper. Therefore, we need to prove the uniformity by asking several annotators to perform the tagging and then evaluating inter-rater reliability. In addition, the size of the log data used in this analysis is limited, as the data comes from the real field event organized by NPO Pangaea, unlike a regular experiment. Therefore, we need to conduct a controlled experiment to validate the findings of this research. This paper, however, makes it possible to produce a facilitation manual for children's workshops. In addition, we plan to develop a facilitator agent based on our findings that helps low-resource language speaking children to actively participate in multilingual communication.

\section{ACKNOWLEDGMENTS}

This research was partially supported by a Grant-in-Aid for Scientific Research (A) (17H00759, 2017-2020), a Grant-in-Aid for Scientific Research (B) $(21 \mathrm{H} 03561,2021-2024)$ and a Grant-in-Aid for Early-Career Scientists (21K17794,2021-2024) from the Japan Society for the Promotion of Sciences(JSPS).

\section{SELECTION AND PARTICIPATION OF CHILDREN}

The children who participated in KISSY were selected from Pangaea members who were significant participants in NPO Pangaea's activity. In addition, some children applied for this summer school via KISSY's Call for Participants web page. All the participants had written parental consent to participate. The participants and parents were informed that the collected data from the participants will be used for academic purposes on the condition that the data was anonymized.

\section{REFERENCES}

[1] Milam Aiken. 2002. Multilingual Communication in electronic Meetings. SIGGROUP Bulletin 23, 1 (2002), 18-19.

[2] Shohei Hida. 2016. Supporting Multi-Language Communication in Children's. Master's thesis. Kyoto University. 
[3] Toru Ishida, Yohei Murakami, Donghui Lin, Takao Nakaguchi, and Masayuki Otani. 2018. Language Service Infrastructure on the Web: The Language Grid. Computer 51, 6 (2018), 72-81. https://doi.org/10.1109/MC.2018.2701643

[4] Mondheera Pituxcoosuvarn, Toru Ishida, Naomi Yamashita, Toshiyuki Takasaki, and Yumiko Mori. 2018. Machine Translation Usage in a Children's Workshop. In Collaboration Technologies and Social Computing, Hironori Egi, Takaya Yuizono, Nelson Baloian, Takashi Yoshino, Satoshi Ichimura, and Armanda Rodrigues (Eds.). Springer International Publishing, Cham, 59-73.

[5] Mondheera Pituxcoosuvarn, Toru Ishida, Naomi Yamashita, Toshiyuki Takasaki, and Yumiko Mori. 2018. Supporting a Children's Workshop with Machine Translation. In Proceedings of the 23rd International Conference on Intelligent User Interfaces Companion (Tokyo, Japan) (IUI '18 Companion). Association for Computing Machinery, New York, NY, USA, Article 32, 2 pages. https: //doi.org/10.1145/3180308.3180341

[6] John R. Searle. 1985. Expression and meaning: Studies in the theory of speech acts. Cambridge University Press.

[7] Yohtaro Takano and Akiko Noda. 1993. A Temporary Decline of Thinking Ability During Foreign Language Processing. Fournal of Cross-Cultural Psychology 24, 4 (1993), 445-462. https://doi.org/10.1177/0022022193244005
arXiv:https://doi.org/10.1177/0022022193244005

[8] Kirsikka Vaajakallio, Jung-Joo Lee, and Tuuli Mattelmäki. 2009. "It Has to Be a Group Work!": Co-Design with Children. In Proceedings of the 8th International Conference on Interaction Design and Children (Como, Italy) (IDC '09). Association for Computing Machinery, New York, NY, USA, 246-249. https://doi.org/10. 1145/1551788.1551843

[9] Daniel Vanderveken. 2009. Meaning and Speech Acts: Volume 1, Principles of Language Use. Cambridge University Press.

[10] Naomi Yamashita, Rieko Inaba, Hideaki Kuzuoka, and Toru Ishida. 2009. Difficulties in Establishing Common Ground in Multiparty Groups Using Machine Translation. In Proceedings of the SIGCHI Conference on Human Factors in Computing Systems (Boston, MA, USA) (CHI '09). Association for Computing Machinery, New York, NY, USA, 679-688. https://doi.org/10.1145/1518701.1518807

[11] Naomi Yamashita and Toru Ishida. 2006. Effects of Machine Translation on Collaborative Work. In Proceedings of the 2006 20th Anniversary Conference on Computer Supported Cooperative Work (Banff, Alberta, Canada) (CSCW '06). Association for Computing Machinery, New York, NY, USA, 515-524. https: //doi.org/10.1145/1180875.1180955 